Proyecciones Journal of Mathematics

Vol. 41, No 1, pp. 83-99, February 2022.

Universidad Católica del Norte

Antofagasta - Chile

\title{
(Completely) weak simple semigroups and (Completely) weak 0-simple semigroups
}

\author{
Leila Nouri \\ University of Sistan and Baluchestan, Iran \\ and \\ Hossein Mohammadzadeh Saany \\ University of Sistan and Baluchestan, Iran \\ Received: March 2020. Accepted : February 2021
}

\begin{abstract}
The structure theorems for (Completely) simple semigroups and (Completely) 0-simple semigroups have proved a powerful tool in the investigation of such semigroups. In this paper, first of all, we define weak simple semigroups and weak 0-simple semigroups and compare them with simple semigroups and 0-simple semigroups respectively. Then we give examples of these semigroups and describe the structure of them. Also, we define completely weak simple semigroup and completely weak 0-simple semigroup. Finally, by using Green's equivalences, we prove some results and give equivalences, for these semigroups.
\end{abstract}

MSC(2010): Primary 20M10; Secondary 20M12.

Keywords: Ideal, (0-) simple semigroups, Completely (0-) simple semigroups, Regular semigroup, Green's equivalences. 


\section{Preliminaries}

This paper is concerned with the study of a certain basic type of semigroup, known as a (completely) weak (0-) simple semigroup. First, we recall from [2] that a semigroup without zero is called simple if it has no proper ideals. A semigroup $S$ with zero ( $S$ should have at least two elements) is called 0 -simple if

(i) $\{0\}$ and $S$ are its only ideals;

(ii) $S^{2} \neq\{0\}$.

For background material on algebraic structures and some other terminologies of these concepts, we refer the reader to $[1,2,3]$.

\section{Weak (0-) Simple Semigroups}

In this section, first of all, we define weak simple semigroups and weak 0simple semigroups and describe the structure of 0-simple semigroup. Then compare weak simple semigroups and weak 0 -simple semigroups with simple semigroups and 0 -simple semigroups, respectively. Then we give examples of weak simple semigroups and weak 0 -simple semigroups. Also, we prove some results about these semigroups.

Definition 2.1. We say that the semigroup $S$ without zero is weak simple if the powers of $S$ are the only ideals of $S$.

Indeed, if $S$ is a weak simple semigroup and $S^{2}=S$, then $S$ is a simple semigroup.

Definition 2.2. We say that the semigroup $S$ with zero is weak 0 -simple, if

(i) $\{0\}$ and the powers of $S$ are the only ideals of $S$;

(ii) $S^{n} \neq\{0\}$, for every $n \geq 2(n \in \mathbf{N})$.

Indeed, if $S$ is a weak 0 -simple semigroup and $S^{2}=S$, then $\mathrm{S}$ is a 0 -simple semigroup. 


\section{Description of the structure of weak 0-simple semigroup:}

Let $S$ is a weak 0-simple semigroup such that $S^{2} \neq S$. Let $\alpha \in S \backslash S^{2}$. It is clear that $S^{2} \cup\{\alpha\}$ is an ideal of $S$ and $S^{2}$ is a proper subset of $S^{2} \cup\{\alpha\}$. Then $S=S^{2} \cup\{\alpha\}$ because $S$ is a weak 0-simple semigroup. Now if $S^{2}=S^{3}$, then the powers of $S$ will be static, for every $n \geq 2$. Otherwise, if $S^{2} \neq S^{3}$, then we let $u \in S^{2} \backslash S^{3}$. We can write $u=x y$, for $x, y \in S$. According to $S=S^{2} \cup\{\alpha\}$, there are three cases that can arise:

(i) $x$ and $y$ belong to $S^{2}$;

(ii) only one of $x$ or $y$ belongs to $S^{2}$ and the other one equals $\alpha$;

(iii) $x=\alpha=y$.

But $(i)$ and (ii) lead to $u \in S^{3}$, which is a contradiction. Thus only case (iii) can occur. So $u=\alpha^{2}$. Now the subset $S^{3} \cup\left\{\alpha^{2}\right\}$ is an ideal of $S$ such that

$$
S^{3} \subseteq S^{3} \cup\left\{\alpha^{2}\right\} \subseteq S^{2} .
$$

Thus $S^{2}=S^{3} \cup\left\{\alpha^{2}\right\}$, and so $S=S^{3} \cup\left\{\alpha, \alpha^{2}\right\}$, such that $\alpha^{i} \in S^{i} \backslash S^{i+1}$, for $i=1,2$. Again if $S^{3}=S^{4}$, then the powers of $S$ will be static, for every $n \geq 3$. Otherwise, if $S^{3} \neq S^{4}$, then in a similar way, we can show $S^{3}=S^{4} \cup\left\{\alpha^{3}\right\}$, and so

$$
S=S^{4} \cup\left\{\alpha, \alpha^{2}, \alpha^{3}\right\},
$$

such that $\alpha^{i} \in S^{i} \backslash S^{i+1}$, for $i=1,2,3$. Therefore by using induction, we have the following theorem.

Theorem 2.3. Let $S$ is a weak 0 -simple semigroup. Then one of the following statements holds:

(i) $S^{2}=S$, and so $S$ is a 0 -simple semigroup.

(ii) There exists $n \geq 2$, such that $S^{n-1} \neq S^{n}$ and $S^{n}=S^{n+1}$. In this case, there exists $\alpha \in S$, such that $S^{i}=S^{i+1} \cup\left\{\alpha^{i}\right\}$, and $\alpha^{i} \in S^{i} \backslash S^{i+1}$, for $1 \leq i \leq n-1$. Also $S=S^{i+1} \cup\left\{\alpha, \alpha^{2}, \ldots, \alpha^{i}\right\}, 1 \leq i \leq n-1$.

(iii) $S^{n} \neq S^{n+1}$, for every $n \geq 1$. In this case, there exists $\alpha \in S$, such that for every $i \geq 1$,

$$
S^{i}=S^{i+1} \cup\left\{\alpha^{i}\right\} .
$$

Thus $S=S^{i+1} \cup\left\{\alpha, \alpha^{2}, \ldots, \alpha^{i}\right\}$, such that $\alpha^{j} \in S^{j} \backslash S^{j+1}, j \in \mathbf{N}$. 
Note that if $S$ is a weak 0 -simple semigroup such that $S^{2} \neq S$, then $\left|S \backslash S^{2}\right|=1$. Since if $\alpha, \beta \in S \backslash S^{2}$, then $S^{2} \cup\{\alpha\}$ and $S^{2} \cup\{\beta\}$ are ideals of $S$ such that $S^{2} \subset S^{2} \cup\{\alpha\}$ and $S^{2} \subset S^{2} \cup\{\beta\}$. Therefore $S^{2} \cup\{\alpha\}=$ $S=S^{2} \cup\{\beta\}$, and so $\alpha=\beta$. By a similar argument, if $S^{n} \neq S^{n+1}$, then $\left|S^{n} \backslash S^{n+1}\right|=1$.

Theorem 2.4. Let $S$ is a weak 0-simple semigroup such that $S^{2} \neq S$. Then $S$ is a monogenic semigroup with zero.

Proof. Since $S^{2} \neq S$, by Theorem 2.3, one of the following cases is held:

Case 1. There exists $n \geq 2$, such that $S^{n-1} \neq S^{n}$ and $S^{n}=S^{n+1}$, that is,

$$
S^{n+1}=S^{n} \subset S^{n-1} \subset \ldots \subset S^{2} \subset S .
$$

Let $S \backslash S^{2}=\{\alpha\}$. Then $S=S^{n} \cup\left\{\alpha, \alpha^{2}, \ldots, \alpha^{n-1}\right\}$. Let $0 \neq x \in S^{m}$, $m \geq n$. Then $x=x_{1} x_{2} \ldots x_{m}$, such that $x_{i} \in S \backslash S^{2}=\{\alpha\}, 1 \leq i \leq m$, that is $x=\alpha^{m}$. Hence

$$
S^{n}=\left\{\alpha^{n}, \alpha^{n+1}, \alpha^{n+2}, \ldots\right\} \cup\{0\}
$$

and

$$
S^{n+1}=\left\{\alpha^{n+1}, \alpha^{n+2}, \alpha^{n+3}, \ldots\right\} \cup\{0\} .
$$

Since $S^{n}=S^{n+1}$, there exits $j \geq 1$ such that $\alpha^{n}=\alpha^{n+j}$. Let $r$ is the smallest natural number such that $\alpha^{n}=\alpha^{n+r}$. Then $S^{n}=\left\{\alpha^{n}, \alpha^{n+1}, \ldots, \alpha^{n+r-1}\right\} \cup$ $\{0\}$, and so

$$
S=\left\{\alpha, \alpha^{2}, \ldots, \alpha^{n}, \alpha^{n+1}, \ldots, \alpha^{n+r-1}\right\} \cup\{0\},
$$

which is a finite monogenic semigroup with zero of index $n$ and period $r=\left|S^{n}\right|$.

Case 2. $S^{n} \neq S^{n+1}$, for every $n \geq 1$. That is,

$$
\ldots \subset S^{n} \subset S^{n-1} \subset \ldots \subset S^{2} \subset S .
$$

In this case, by Theorem 2.3(iii), there exists $\alpha \in S$ such that $S=S^{i+1} \cup$ $\left\{\alpha, \alpha^{2}, \ldots, \alpha^{i}\right\}$, for every $i \geq 1$, which implies $S$ is an infinite monogenic semigroup with zero.

From Theorems 2.3 and 2.4, we have the following corollary. 
Corollary 2.5. For a semigroup $S$ the following statements are equivalent:

(i) $S$ is a weak 0-simple semigroup such that is not 0-simple;

(ii) $S$ is an infinite monogenic semigroup with zero or is a finite monogenic semigroup with zero of index $n(n>1)$ and period $r$.

Now we give examples of weak simple semigroups and weak 0 -simple semigroups.

Example 2.6. Let $S=\langle a\rangle=\left\{a^{i} \mid i \geq 1\right\}$, is an infinite monogenic semigroup. Then only ideals of $S$, are powers of $S$, and so $S$ is a weak simple semigroup. Also

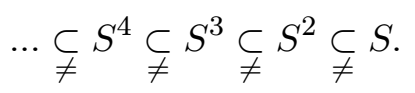

That is, the chain of subsemigroups of $S$ is not static.

If we add zero to the above infinite monogenic semigroup, then the made semigroup is weak 0 -simple, such that the chain of subsemigroups of $S$ is not static.

Example 2.7. Let $S=<b>=\left\{b, b^{2}, \ldots, b^{m}, b^{m+1}, \ldots, b^{m+r-2}, b^{m+r-1}\right\}$, is a finite monogenic semigroup, of index $m$, and period $r$. Obviously,

$$
\begin{aligned}
& S^{2}=<b>^{2}=\left\{b^{2}, b^{3}, \ldots, b^{m}, b^{m+1}, \ldots, b^{m+r-2}, b^{m+r-1}\right\}, \\
& S^{3}=<b>^{3}=\left\{b^{3}, \ldots, b^{m}, b^{m+1}, \ldots, b^{m+r-2}, b^{m+r-1}\right\}, \\
& \vdots \\
& S^{m}=<b>^{m}=\left\{b^{m}, b^{m+1}, \ldots, b^{m+r-2}, b^{m+r-1}\right\}, \\
& S^{m+1}=S^{m} .
\end{aligned}
$$

Thus $S^{m+1}=S^{m} \subsetneq S^{m-1} \varsubsetneqq \ldots \underset{f}{\subsetneq} S^{2} \varsubsetneqq S$. Also every ideal of $S$ is a power of $S$. Therefore $S$ is a weak simple semigroup, such that the powers of $S$ will be static, for every $k \geq m$. In this case, $S^{m}$ is a group.

Notice that the converse of Theorem 2.3 is not true in general, that is, if there exists a semigroup $S$ with zero, such that satisfies in condition (ii) or condition (iii) of Theorem 2.3, then $S$ necessity is not weak 0 -simple semigroup. See the following example. 
Example 2.8. Let $B=\left\{\beta_{1}, \beta_{2}, \ldots\right\}$, that can be finite or infinite. Define:

$$
\begin{aligned}
& \beta_{i} \cdot \beta_{i}=\beta_{i}^{2}=\beta_{i}, \quad \forall i \geq 1 ; \\
& \beta_{i} \cdot \beta_{k}=\beta_{k} \cdot \beta_{i}=\beta_{i}, \quad \forall i, k \in \mathbf{N}, i<k .
\end{aligned}
$$

Obviously, $B$ is a semigroup. Now let $T$ is a weak 0-simple semigroup, which $\theta$ is its zero. Consider $S=T \cup B$, with the following operation:

If two elements are in $T$ or $B$, then the operation is the same operation $T$ and $B$ respectively. Also,

$$
\begin{aligned}
& \beta_{i} . t=t . \beta_{i}=\beta_{i}, \quad \forall \beta_{i} \in B, \forall t \in T, t \neq \theta ; \\
& \beta_{i} \cdot \theta=\theta \cdot \beta_{i}=\theta, \quad \forall \beta_{i} \in B .
\end{aligned}
$$

Clearly, $S$ is a semigroup, such that $S^{m}=T^{m} \cup B$, for every $m \geq 1$. Since $T$ is a weak 0 -simple semigroup, there exists $\alpha$ in $T$, such that $T=$ $T^{2} \cup\{\alpha\}$. Therefore

$$
S=T \cup B=S^{2} \cup\{\alpha\},
$$

which $\alpha \notin S^{2}$. Now if $T$ is a semigroup as Example 2.6, which the chain of subsemigroups of $T$ is not static, then we have $S^{i}=S^{i+1} \cup\left\{\alpha^{i}\right\}$, for every $i \geq 1$. Then

$$
S=S^{i+1} \cup\left\{\alpha^{1}, \alpha^{2}, \ldots, \alpha^{i}\right\}, \quad \alpha^{j} \in S^{j} \backslash S^{j+1}, \quad \forall j \geq 1 .
$$

Therefore $S$ satisfies in condition (iii) of Theorem 2.3, but $S$ is not weak 0 -simple semigroup, because $B \cup\{\theta\}$ is an ideal of $S$, which is not as a power of $S$.

Also if $T$ is a semigroup as Example 2.7, which the chain of subsemigroups of $T$ is static, then $S$ satisfies in condition (ii) of Theorem 2.3, but $S$ is not weak 0-simple semigroup, because $B \cup\{\theta\}$ is an ideal of $S$, which is not as a power of $S$.

Recall from [2] that an equivalence $\mathcal{J}$ on $S$ is defined by the rule that $a \mathcal{J} b$, for $a, b \in S$, if and only if $S^{1} a S^{1}=S^{1} b S^{1}$. Also, the $\mathcal{J}$-class containing the element $a$ is denoted by $J_{a}$. 
Theorem 2.9. Let $S$ is a weak 0 -simple semigroup. Then one of the following statements holds:

(i) $J_{a}=\{a\}$, for every $a \in S$.

(ii) There exists $n \geq 1$ such that for every $a, b \in S^{n} \backslash\{0\}, a \mathcal{J} b$ and for every $a \in S \backslash S^{n}, J_{a}=\{a\}$.

Proof. By Theorem 2.3, one of the following cases holds:

Case 1. $S^{2}=S$, and so $S$ is a 0 -simple. Since $S^{1} a S^{1}$ is a non zero ideal of $S$, for every $a \in S \backslash\{0\}, S^{1} a S^{1}=S$, and so $a \mathcal{J} b$, for every $a, b \in S^{2} \backslash\{0\}=S \backslash\{0\}$. In this case $S \backslash S^{2}=\emptyset$. Hence (ii) is satisfied.

Case 2. There exists $n \geq 2$, such that $S^{n-1} \neq S^{n}$ and $S^{n}=S^{n+1}$, that is,

$$
S^{n+1}=S^{n} \subset S^{n-1} \subset \ldots \subset S^{2} \subset S .
$$

Now $S^{1} a S^{1} \subseteq S^{1} S^{n} S^{1} \subseteq S^{n}$, for every $a \in S^{n} \backslash\{0\}$, and so by assumption $S^{1} a S^{1}=S^{n}$. Therefore $a \mathcal{J} b$, for every $a, b \in S^{n} \backslash\{0\}$. Also $S \backslash S^{n}=\left\{\alpha, \alpha^{2}, \ldots, \alpha^{n-1}\right\}$, such that $\alpha^{i} \in S^{i} \backslash S^{i+1}, 1 \leq i \leq n-1$. Hence $S^{1} \alpha^{i} S^{1} \neq S^{1} \alpha^{j} S^{1}$, for every $1 \leq i \neq j \leq n-1$, and so $\alpha^{i} \mathcal{J} \alpha^{j}$, for every $1 \leq i \neq j \leq n-1$. Therefore $J_{a}=\{a\}$, for every $a \in S \backslash S^{n}$, and so (ii) is satisfied.

Case 3. $S^{n} \neq S^{n+1}$, for every $n \geq 1$. That is,

$$
\ldots \subset S^{n} \subset S^{n-1} \subset \ldots \subset S^{2} \subset S .
$$

In this case, by Theorem 2.3(iii), $S$ is an infinite monogenic semigroup with zero, and so $S^{1} a S^{1} \neq S^{1} b S^{1}$, for every $a \neq b \in S$, that is $J_{a}=\{a\}$, for every $a \in S$. Therefore $(i)$ is satisfied.

The converse of Theorem 2.9 is not true, see the following example.

Example 2.10. Consider $S$ with the following table: 


\begin{tabular}{c|cccc} 
& 0 & $\mathrm{a}$ & $\mathrm{b}$ & $c$ \\
\hline 0 & 0 & 0 & 0 & 0 \\
$a$ & 0 & $b$ & 0 & 0 \\
$b$ & 0 & 0 & $\mathrm{a}$ & 0 \\
$c$ & 0 & 0 & 0 & 0
\end{tabular}

Indeed, $S^{2}=\{0, a, b\}, a \mathcal{J} b$ and $J_{c}=\{c\}$. Therefore condition (ii) of Theorem 2.9, for $n=2$ is satisfied. But $I=\{0, c\}$ is a non zero ideal of $S$, which is not equals with no powers of $S$, because $S^{n}=S^{n+1}$, for every $n \geq 2$.

Theorem 2.11. Let $S$ is a weak 0 -simple semigroup. Then for every $0 \neq$ $a \in S$, there exists $m \geq 1$, such that $S a S=S^{m}$.

Proof. Let $0 \neq a \in S$. If $S a S=\{0\}$, then $I=\{\alpha \mid S \alpha S=\{0\}\}$ is a nonzero ideal of $S$. Since $S$ is a weak 0 -simple semigroup, there exists $n \geq 1$, such that $I=S^{n}$. But $S I S=\{0\}$, by definition $I$. Then $S^{n+2}=\{0\}$, which is a contradiction. Therefore $S a S \neq\{0\}$. Since $S a S$ is an ideal of $S$, there exists $m \geq 1$, such that $S a S=S^{m}$.

Theorem 2.12. The semigroup $S$ is weak 0 -simple if and only if for every $n \geq 1, S^{n} \neq\{0\}$, and for every $0 \neq a \in S$ there exists $m \geq 1$, such that $S a S=S^{m}$.

Proof. Necessity. It is obvious by definition and Theorem 2.11.

Sufficiency. Let $I$ is a non zero ideal of $S$. Then for every $0 \neq a \in I$, there exists $m_{a} \in \mathbf{N}$, such that $S^{1} a S^{1}=S^{m_{a}}$. Therefore $I=\cup_{a \in I} S^{m_{a}}$. Let $m=\min \left\{m_{a} \mid a \in I\right\}$. Then $I=S^{m}$, and so $S$ is a weak 0 -simple semigroup.

Lemma 2.13. Let $S$ is a semigroup with zero, such that for every $0 \neq$ $a \in S$, there exists $m \geq 1$, such that $S a S=S^{m}$. If $A$ is a non-empty and non-zero subset of $S$, then there exists $m \geq 1$, such that $S A S=S^{m}$.

Proof. By assumption we have for every $0 \neq a \in A$, there exists $m_{a} \geq 1$, such that $S a S=S^{m_{a}}$. Thus $S A S=\cup_{a \in A} S a S=\cup_{0 \neq a \in A} S^{m_{a}}$. Let $m=$ $\min \left\{m_{a} \mid a \in A\right\}$. Then $\cup_{0 \neq a \in A} S^{m_{a}}=S^{m}$, and so $S A S=S^{m}$, as required. 
Theorem 2.14. Let $S$ is a weak 0 -simple semigroup, such that $S^{n+1}=S^{n}$, for $n \geq 1$. Then $S^{n}$ is a 0 -simple semigroup.

Proof. We must show the only non-zero ideal of $S^{n}$ is $S^{n}$. Let $A$ is a non-zero ideal of $S^{n}$. Since $S$ is a weak 0-simple semigroup, by Theorem 2.11 , for every $0 \neq a \in S$ there exists $m \geq 1$, such that $S a S=S^{m}$. Thus $S A S=S^{k}$, for $k \geq 1$, by Lemma 2.13. Therefore $S^{n} A S^{n}=S^{k+2 n-2}$. Since $k+2 n-2 \geq n$, from equality $S^{n}=S^{n+1}$, we obtain $S^{n} A S^{n}=S^{k+2 n-2}=$ $S^{n}$. But $S^{n}=S^{n} A S^{n} \subseteq A$, because $A$ is an ideal of $S$. Thus $S^{n}=A$, that is, the only non-zero ideal of $S^{n}$ is $S^{n}$, and so $S^{n}$ is a 0 -simple semigroup, as required.

Notice that, since in a finite weak 0-simple semigroup, the chain of powers of $S$ is static, thus in these semigroups there exists a power of $S$, such that is a 0 -simple semigroup.

For a semigroup $S$, the set of all idempotents of $S$ is denoted by $E(S)$.

Lemma 2.15. Let $S$ is a weak 0 -simple semigroup, such that $E(S) \neq\{0\}$. Let $S e S=S^{m}$, for $0 \neq e \in E(S)$ and $m \in \mathbf{N}$. Then the chain of powers of $S$ is static, for every $k \geq m$, and so $S^{m}$ is 0 -simple.

Proof. Since $S$ is a weak 0 -simple semigroup, for $0 \neq e \in E(S)$ there exists $m \geq 1$, such that $S e S=S^{m}$, by Theorem 2.11. Indeed $e \in S^{n}$, for every $n \geq 1$. Then $S^{m}=S e S \subseteq S^{k}$, for every $k \geq 3$. If $m=1$ or $m=2$, then obviously the converse of inclusion is satisfied, and so $S^{m}=S^{k}$, for every $k \geq 3$. Thus $S^{m}=S^{m+1}$. Now if $m \geq 3$, since above inclusion is satisfied, for every $k \geq 3$, thus for $k=m+1$, is satisfied too, that is $S^{m} \subseteq S^{m+1}$, and so $S^{m}=S^{m+1}$. Therefore the chain of powers of $S$ is static, for every $k \geq m$, and so $S^{m}$ is 0 -simple, by Theorem 2.14 .

Lemma 2.16. Let $S$ is a weak 0 -simple semigroup, such that $E(S) \neq\{0\}$. Let $S E(S) S=S^{m}$, for $m \in \mathbf{N}$. Then the chain of powers of $S$ is static, for every $k \geq m$, and so $S^{m}$ is 0 -simple.

Proof. Since $S$ is a weak 0-simple semigroup, there exists $m \geq 1$ such that $S E(S) S=S^{m}$, by Lemma 2.13. Since $E(S) \subseteq S^{n}$, for every $n \geq 1$, therefore $S^{m}=S E(S) S \subseteq S^{k}$, for every $k \geq 3$. Now by using a similar argument as in the proof of Lemma 2.15, we obtain the result. 
Recall from [2] that an element $a$ of semigroup $S$ is called regular if $a=a x a$, for some $x \in S$. If all elements of $S$ are regular, we say that $S$ is a regular semigroup.

Corollary 2.17. Every regular weak 0 -simple semigroup is a 0 -simple semigroup.

Proof. Let $S$ is a regular weak 0-simple semigroup. Then for every $a \in S$, there exists $x \in S$, such that $a=a x a$, and so $a=a(x a)(x a)$. Since $x a \in E(S)$, therefore $a=a(x a)(x a) \in S E(S) S$, and so $S \subseteq S E(S) S$. Obviously, the converse of inclusion is satisfied. Thus $S=S E(S) S$, and so $S$ is 0 -simple, by Lemma 2.16 , as required.

\section{Completely Weak (0-) Simple Semigroups}

In this section by using the natural partial order relation on the idempotents of a semigroup, we define completely weak simple semigroups and completely weak 0 -simple semigroups. Then we prove some results of these semigroups and give equivalences of them.

Among idempotents in an arbitrary semigroup, there is a natural partial order relation defined by the rule that $e \leq f$ if and only if $e f=f e=e$. It is easy to verify that the given relation is a partial order relation. If $S$ is a semigroup with zero, then the defining properties of a zero element immediately imply that 0 is the unique minimum idempotent. The idempotents that are minimal within the set of non-zero idempotents are called primitive. Thus a primitive idempotent $e$ has the property that

$$
e f=f e=f \neq 0 \Longrightarrow e=f .
$$

Recall from [2] that the semigroup $S$ is called completely simple if it is simple and $(E(S), \leq)$ has a primitive idempotent. Also, the semigroup Siscalledcompletely0-simpleifitis0-simpleand $(\mathrm{E}(\mathrm{S}), \leq)$ has a primitive idempotent.

Definition 3.1. We say that the semigroup $S$ is completely weak simple if $S$ is a weak simple and $(E(S), \leq)$ has a primitive idempotent.

Definition 3.2. We say that the semigroup $S$ is completely weak 0-simple if $S$ is a weak 0 -simple and $(E(S), \leq)$ has a primitive idempotent. 
Since in every finite weak simple semigroup, every element has a power which is idempotent, $E(S) \neq \emptyset$. Also since $|E(S)|<\infty, E(S)$ has a primitive idempotent. Therefore every finite weak simple semigroup is completely weak simple.

In every finite weak 0-simple semigroup, if $E(S) \neq\{0\}$, then by using a similar argument as in the finite weak simple semigroup, we can conclude that every finite weak 0 -simple semigroup is completely weak 0 -simple.

Notice that if $S$ is a completely weak 0-simple semigroup, then $S$ is not an infinite monogenic semigroup. Now similar to Theorem 2.3 we have the following theorem.

Theorem 3.3. Let $S$ is a completely weak 0 -simple semigroup. Then one of the following statements holds:

(i) $S^{2}=S$, and so $S$ is a completely 0-simple semigroup.

(ii) There exists $n \geq 2$, such that $S^{n-1} \neq S^{n}$ and $S^{n}=S^{n+1}$. In this case, $S^{n} \backslash\{0\}$ is a group, and $S^{n}$ is a completely 0-simple semigroup.

Recall from [2] that an equivalence $\mathcal{L}$ on $S$ is defined by the rule that $a \mathcal{L} b$, for $a, b \in S$, if and only if $S^{1} a=S^{1} b$. Similarly, an equivalence $\mathcal{R}$ on $S$ is defined by the rule that $a \mathcal{R} b$, for $a, b \in S$, if and only if $a S^{1}=b S^{1}$, and the join $\mathcal{L} \vee \mathcal{R}$ is denoted by $\mathcal{D}$. Also the $\mathcal{L}$-class [R-class, $\mathcal{D}$-class] containing the element $a$ is denoted by $L_{a}\left[R_{a}, D_{a}\right]$.

Lemma 3.4. Let $S$ is a completely weak 0-simple semigroup and $e$ is a primitive idempotent. Then,

(i) $R_{e}=e S \backslash\{0\}$

(ii) $L_{e}=S e \backslash\{0\}$.

Proof. We prove $(i)$, the proof $(i i)$ is similar. If $a \in R_{e}$, it is clear that $a \neq 0$. Since $e$ is a left identity element for $R_{e}, e a=a \in e S \backslash\{0\}$, and so $R_{e} \subseteq e S \backslash\{0\}$. Now let $a=e s \in e S \backslash\{0\}$. Then $e a=e^{2} s=e s=a$. Since $S$ is a weak 0 -simple semigroup, there exist $t, z \in S$, such that $e=z a t$, by Theorem 2.11. Now by using a similar argument as in the proof of $[2$, Lemma 3.2.4], we obtain the result. 
Lemma 3.5. Let $S$ is a completely weak 0 -simple semigroup. Then there exists $n \geq 1$, such that for every $a_{1}, a_{2}, \ldots, a_{n} \in S$, if $a_{1} a_{2} \ldots a_{n} \neq 0$, then

(i) $R_{a_{1} a_{2} \ldots a_{n}}=\left(a_{1} a_{2} \ldots a_{n} S\right) \backslash\{0\}$.

(ii) $L_{a_{1} a_{2} \ldots a_{n}}=\left(S a_{1} a_{2} \ldots a_{n}\right) \backslash\{0\}$.

Proof. (i) Let $e$ is a primitive idempotent. Then there exists $n \geq 1$, such that $S e S=S^{n}$, by Theorem 2.11. Now let $a_{1}, a_{2}, \ldots, a_{n} \in S$, such that $a_{1} a_{2} \ldots a_{n} \neq 0$ and $u \in\left(a_{1} a_{2} \ldots a_{n} S\right) \backslash\{0\}$. Since $S e S=S^{n}$, there exist $z, t \in S$, such that $a_{1} a_{2} \ldots a_{n}=z e t$, and so for some $v \in S, u=z e v$. Thus $e \mathcal{R} e t$ and $e \mathcal{R} e v$, by Lemma 3.4, and so ev Ret. Since $\mathcal{R}$ is a left congruence, we obtain zevRzet, that is $u \mathcal{R} a_{1} a_{2} \ldots a_{n}$, and so $u \in R_{a_{1} a_{2} \ldots a_{n}}$. Therefore $\left(a_{1} a_{2} \ldots a_{n} S\right) \backslash\{0\} \subseteq R_{a_{1} a_{2} \ldots a_{n}}$, hence

$$
\left[\left(a_{1} a_{2} \ldots a_{n} S\right) \backslash\{0\}\right] \backslash\left\{a_{1} a_{2} \ldots a_{n}\right\} \subseteq\left[R_{a_{1} a_{2} \ldots a_{n}} \backslash\left\{a_{1} a_{2} \ldots a_{n}\right\}\right] .
$$

Now let $c \in R_{a_{1} a_{2} \ldots a_{n}} \backslash\left\{a_{1} a_{2} \ldots a_{n}\right\}$. Then there exists $x \in S$, such that $c=a_{1} a_{2} \ldots a_{n} x$. Thus $c \in\left[\left(a_{1} a_{2} \ldots a_{n} S\right) \backslash\{0\}\right] \backslash\left\{a_{1} a_{2} \ldots a_{n}\right\}$, and so

$$
R_{a_{1} a_{2} \ldots a_{n}} \backslash\left\{a_{1} a_{2} \ldots a_{n}\right\} \subseteq\left[\left(a_{1} a_{2} \ldots a_{n} S\right) \backslash\{0\}\right] \backslash\left\{a_{1} a_{2} \ldots a_{n}\right\} .
$$

Therefore $R_{a_{1} a_{2} \ldots a_{n}} \backslash\left\{a_{1} a_{2} \ldots a_{n}\right\}=\left[\left(a_{1} a_{2} \ldots a_{n} S\right) \backslash\{0\}\right] \backslash\left\{a_{1} a_{2} \ldots a_{n}\right\}$, by (1) and (2). Hence $R_{a_{1} a_{2} \ldots a_{n}}=a_{1} \ldots a_{n} S \backslash\{0\}$, as required.

(ii) It is similar to the proof of $(i)$.

Theorem 3.6. Let $S$ is a completely weak 0 -simple semigroup. Then there exists $n \geq 1$, such that $S^{n}$ is completely 0 -simple.

Proof. Since $S$ is a completely weak 0-simple semigroup, by Lemma 3.5, there exists $n \geq 1$, such that for every $a_{1}, a_{2}, \ldots, a_{n} \in S$, if $a_{1} a_{2} \ldots a_{n} \neq 0$, then

(i) $R_{a_{1} a_{2} \ldots a_{n}}=\left(a_{1} a_{2} \ldots a_{n} S\right) \backslash\{0\}$.

(ii) $L_{a_{1} a_{2} \ldots a_{n}}=\left(S a_{1} a_{2} \ldots a_{n}\right) \backslash\{0\}$.

Let $x=a_{1} \ldots a_{n}$ and $y=b_{1} \ldots b_{n}$ are a non-zero elements of $S^{n}$. Then $a_{1} \ldots a_{n} S b_{1} \ldots b_{n} \neq\{0\}$, since otherwise, if $a_{1} \ldots a_{n} S b_{1} \ldots b_{n}=\{0\}$, then, by 
Theorem 2.11, there exist $m_{1}, m_{2} \in \mathbf{N}$, such that $S a_{1} \ldots a_{n} S=S^{m_{1}}$ and $S b_{1} \ldots b_{n} S=S^{m_{2}}$. Then

$$
\begin{gathered}
S^{m_{1}+m_{2}}=\left(S a_{1} \ldots a_{n} S\right)\left(S b_{1} \ldots b_{n} S\right)=S\left(a_{1} \ldots a_{n} S^{2} b_{1} \ldots b_{n}\right) S \\
\subseteq S\left(a_{1} \ldots a_{n} S b_{1} \ldots b_{n}\right) S=\{0\},
\end{gathered}
$$

and so $S^{m_{1}+m_{2}}=\{0\}$, which is a contradiction. Thus $a_{1} \ldots a_{n} S b_{1} \ldots b_{n} \neq\{0\}$, and so there exists $u \in S$, such that

$$
\left(a_{1} \ldots a_{n}\right) u\left(b_{1} \ldots b_{n}\right)=d \neq 0 .
$$

Since $R_{b_{1} b_{2} \ldots b_{n}}=\left(b_{1} \ldots b_{n} S\right) \backslash\{0\}$, there exists $k \in S$, such that $b_{1} \ldots b_{n} k=$ $b_{1} \ldots b_{n}$. Thus $b_{1} \ldots b_{n} k^{m}=b_{1} \ldots b_{n}$, for every $m \geq 1$. Also since $R_{a_{1} a_{2} \ldots a_{n}}=$ $\left(a_{1} a_{2} \ldots a_{n} S\right) \backslash\{0\}$ and $d \in\left(a_{1} a_{2} \ldots a_{n} S\right) \backslash\{0\}$, there exists $l \in S$, such that

$$
a_{1} \ldots a_{n}=d l=\left(a_{1} \ldots a_{n}\right) u\left(b_{1} \ldots b_{n}\right) l=\left(a_{1} \ldots a_{n}\right) u\left(b_{1} \ldots b_{n}\right) k^{n} l=d k^{n} l .
$$

Therefore $\left(a_{1} \ldots a_{n}, d\right) \in \mathcal{R}^{S^{n}}$. Similarly, we can show $\left(d, b_{1} \ldots b_{n}\right) \in \mathcal{L}^{S^{n}}$, and so

$$
\left(a_{1} \ldots a_{n}, b_{1} \ldots b_{n}\right) \in \mathcal{L}^{S^{n}} \circ \mathcal{R}^{S^{n}}=\mathcal{D}^{S^{n}} .
$$

Thus $S^{n} \backslash\{0\}$ and $\{0\}$ are $\mathcal{D}^{S^{n}}$-classes, in $S^{n}$. Since $E(S) \backslash\{0\} \subseteq S^{n}\{0\}$ and $S$ has a primitive idempotent, $\mathcal{D}^{S^{n}}$-class $S^{n} \backslash\{0\}$ has a regular element, and so $S^{n} \backslash\{0\}$ is regular. Since $\{0\}$ is regular, t $S^{n}$ is regular. Let $a \in S^{n}$. Then there exists $x \in S^{n}$, such that $a=a x a$, and so $a=a(x a)(x a)$. Since $x a \in E(S)$, therefore $a=a(x a)(x a) \in S^{n} E(S) S^{n}$, and so $S^{n} \subseteq S^{n} E(S) S^{n}$. Obviously, the converse of inclusion is satisfied. Thus $S^{n}=S^{n} E(S) S^{n}$, and so $S^{n}=S^{n+1}$, that is $S^{n}$ is 0-simple. Since $S$ has a primitive idempotent and $E(S) \subseteq S^{n}, S^{n}$ is completely 0-simple.

By using a similar argument as in the proof of Theorem 3.6, for every completely weak simple semigroup $S$, there exists $n \geq 1$, such that $S^{n}$ is completely simple and $E(S) \subseteq S^{n}$. Since every idempotent is primitive, in every completely simple semigroup, thus every idempotent is primitive, in every completely weak simple semigroup.

Lemma 3.7. For a regular semigroup $S$, the following statements are equivalent:

(i) $S$ has only one idempotent;

(ii) $S$ is cancellative;

(iii) $S$ is a group. 
Proof. $\quad(i) \Rightarrow(i i)$. Since $S$ is regular, for every $a \in S$, there exists $x \in S$, such that $a=a x a$. Then $a x$ and $x a$ are idempotent. Thus for every $a \in S$, there exists $x \in S$ such that $a x=x a=e$ and $a e=e a=a$. Now let $a c=b c$, for $a, b, c \in S$. Thus there exists $x \in S$ such that $c x=e$. Therefore $a c x=b c x$ implies that $a=b$. Similarly, $c a=c b$ implies that $a=b$, and so $S$ is cancellative, as required.

$($ ii $) \Rightarrow($ iii $)$. Since $S$ is regular and cancellative, for every $a \in S$, there exists $x \in S$ such that $a x a=a$ and $x a x=x$. Let $x a=e$. Then $x a x=x$ implies that $x a x e=x e$, and so $a x=e$. Therefore $a x=x a=e$, and so $a^{2} x=a$ and $a x^{2}=x$. Now $a^{2} x b=a b$, implies that $a(x b)=b$, for every $b \in S$. Also $b a x^{2}=b x$ implies that $b a x=b$, and so $(b x) a=b$, for every $b \in S$. Therefore $a S=S a=S$, for every $a \in S$, that is $S$ is a group.

$($ iii $) \Rightarrow(i)$. It is obvious.

Corollary 3.8. (i) Let $S$ is a weak simple semigroup, such that $|E(S)|=1$. Then there exists $n \geq 1$, such that $S^{n}$ is a group.

(ii) Let $S$ is a completely weak simple semigroup, such that is cancellable. Then there exists $n \geq 1$, such that $S^{n}$ is a group.

Proof. $\quad(i)$. Let $S$ is a weak simple semigroup, such that $|E(S)|=1$. Then $S$ has a primitive element, and so $S$ is a completely weak simple semigroup. Since Theorem 3.6 is also true, for every completely weak simple semigroup, therefore there exists $n \geq 1$, such that $S^{n}$ is completely simple, and so $S^{n}$ is regular. Since $E(S) \subseteq S^{n}$ and $|E(S)|=1, S^{n}$ is a group, by Lemma 3.7.

(ii). Since Theorem 3.6 is also true, for every completely weak simple semigroup, therefore there exists $n \geq 1$, such that $S^{n}$ is completely simple, and so $S^{n}$ is regular. Since $S$ is cancellative, $S^{n}$ is also cancellative, and so $S^{n}$ is a group, by Lemma 3.7 .

Notice that every theorem that said for (completely) weak 0-simple semigroup, satisfies for (completely) weak simple semigroup, too.

Recall from [2] that for $a$ and $b$ in semigroup $S, L_{a} \leq L_{b}\left(R_{a} \leq R_{b}\right)$ if $S^{1} a \subseteq S^{1} b\left(a S^{1} \subseteq b S^{1}\right)$. Thus we may regard $S / \mathcal{L}(S / \mathcal{R})$ as a partially ordered set. The semigroup $S$ satisfies condition $\min _{L}\left(\min _{R}\right)$ according to the partially ordered set $S / \mathcal{L}(S / \mathcal{R})$ satisfies the minimal condition. 
In a semigroup $S$, an ideal minimal within the set of all non-zero ideals is called 0-minimal.

A semigroup $S$ is called group-bound if every element $a$ in $S$ has a power $a^{n}(n \geq 1)$ lying in a subgroup of $S$.

Theorem 3.9. Let $S$ is a weak 0-simple semigroup. Then the following statements are equivalent:

(i) $S$ is a completely weak 0 -simple semigroup;

(ii) there exists $n \geq 1$, such that $S^{n}$ is completely 0-simple;

(iii) there exists $n \geq 1$, such that $S^{n}$ is group-bound and simple;

(iv) there exists $n \geq 1$, such that $S^{n}$ is simple and satisfies in conditions $\min _{R^{S^{n}}}$ and $\min _{L^{S^{n}}}$

$(v)$ there exists $n \geq 1$, such that $S^{n}$ is simple and contains at least one left 0-minimal ideal that is simple and contains at least one right 0 -minimal ideal that is simple.

Proof. $\quad(i) \Rightarrow(i i)$. Since $S$ is a weak 0-simple semigroup, there exists $n \geq 1$, such that $S^{n}$ is completely 0 -simple, by Theorem 3.6, and so (ii) is satisfied.

$($ ii $) \Rightarrow(i)$. Since $E(S) \subseteq S^{n}$, for every $n \geq 1, S$ has a primitive idempotent, and so $S$ completely weak 0 -simple, as required.

Statements (ii), (iii), (iv) and $(v)$ are equivalent, by [2, Theorem 3.2.11].

A semigroup $S$ is called completely regular if every element $a$ of $S$ lies in a subgroup of $S$ (see [2]).

Similarly, we can show the following theorem for weak simple semigroup.

Theorem 3.10. Let $S$ is a weak simple semigroup. Then the following statements are equivalent:

(i) $S$ is a completely weak simple semigroup;

(ii) there exists $n \geq 1$, such that $S^{n}$ is completely simple;

(iii) there exists $n \geq 1$, such that $S^{n}$ is completely regular and simple; 
(iv) there exists $n \geq 1$, such that $S^{n}$ is simple and satisfies in conditions $\min _{R^{S^{n}}}$ and $\min _{L^{S^{n}}}$

(v) there exists $n \geq 1$, such that $S^{n}$ is simple and contains at least one left minimal ideal that is simple and contains at least one right minimal ideal that is simple.

Recall from [2] that a semigroup $S$ has the weak cancelation property if for all $a, b, c$ in $S, c a=c b$ and $a c=b c$ imply that $a=b$.

Theorem 3.11. Let $S$ is a semigroup, such that if $A^{n}=S^{n}$, for ideal $A$ of $S$ and $n \geq 1$, then there exists $m \geq 1$, such that $A=S^{m}$. Then the following statements are equivalent:

(i) $S$ is a completely weak simple semigroup;

(ii) there exists $n \geq 1$, such that $S^{n}$ is completely simple;

(iii) there exists $n \geq 1$, such that $S^{n}$ is regular and has the weak cancelation property;

(iv) there exists $n \geq 1$, such that $S^{n}$ is regular and for every $a, b \in S^{n}$, $a b a=a$ implies $b a b=b$;

(v) there exists $n \geq 1$, such that $S^{n}$ is regular and every idempotent in $S$ is primitive.

Proof. Statements $(i i),(i i i),(i v)$ and $(v)$ are equivalent, by [2, Theorem $3.2 .11]$.

$(i) \Rightarrow(i i)$. Since Theorem 3.6 is also true, for every completely weak simple semigroup, therefore there exists $n \geq 1$, such that $S^{n}$ is completely simple, as required.

$($ ii $) \Rightarrow(i)$. It is sufficient to show $S$ is weak simple. Let $A$ is an ideal of $S$. Then $S^{n} A^{n} S^{n} \subseteq A^{n}$, that is $A^{n}$ is an ideal of $S^{n}$. Thus $A^{n}=S^{n}$, because $S^{n}$ is simple. Now by assumption, there exists $m \geq 1$, such that $A=S^{m}$. Thus the powers of $S$, are the only ideals of $S$, and so $S$ is weak simple.

Theorem 3.12. Let $S$ is a semigroup, such that if $A^{n}=S^{n}$, for ideal $A$ of $S$ and $n \geq 1$, then there exists $m \geq 1$, such that $A=S^{m}$. Then the following statements are equivalent: 
(i) $S$ is a completely weak simple semigroup;

(ii) there exists $n \geq 1$, such that $S^{n}$ is completely simple;

(iii) there exists $n \geq 1$, such that $S^{n}$ is completely regular and for every $x, y \in S^{n}, x x^{-1}=(x y x)(x y x)^{-1}$;

(iv) there exists $n \geq 1$, such that $S^{n}$ is completely regular and simple.

Proof. $\quad(i) \Leftrightarrow(i i)$ is satisfied, by Theorem 3.11.

Statements (ii), (iii) and (iv) are equivalent, by [2, Proposition 4.1.2].

\section{References}

[1] P. M. Higgins, Techniques of semigroup theory. Oxford: Oxford University, 1992.

[2] J. M. Howie, Fundamentals of semigroup theory. London: Oxford University Press, 1995.

[3] M. Kilp, U. Knauer and A. Mikhalev, Monoids, Acts and Categorie. Berlin: Walter de Gruyter, 2000.

\section{Leila Nouri}

Department of Mathematics,

University of Sistan and Baluchestan,

Zahedan,

Iran

e-mail: Leila_Nouri@math.usb.ac.ir

and

\section{Hossein Mohammadzadeh Saany}

Department of Mathematics,

University of Sistan and Baluchestan,

Zahedan,

Iran

e-mail: hmsdm@math.usb.ac.ir

Corresponding author 